\title{
The Risk-based Treatment of Acute Pulmonary Embolism
}

\author{
Luca Masottia, h, Annalisa Mannuccia, Fabio Antonelli ${ }^{\mathrm{b}}$, Vincenzo Maurinic, \\ Roberto Testa $^{\mathrm{d}}$, Sergio Marchetti ${ }^{\mathrm{e}}$, Giancarlo Landiniif ${ }^{\mathrm{f}}$, Roberto Cappelli ${ }^{\mathrm{g}}$
}

\begin{abstract}
Risk evaluation and prognostic stratification based upon clinical and radiological findings and new cardiac biomarkers, such as natriuretic peptides (NP) and troponins, represent key points in modern management of acute pulmonary embolism (PE). Literature evidence shows that normotensive PE with right heart dysfunction (RHD), defined as submassive PE, has poorer prognosis when compared to normotensive PE without RHD, defined as non-massive PE; thus whether submassive PE should be managed more aggressively and with closer monitoring represents the crucial question about acute PE treatment. Although the answer is yet unclear, the most recent guidelines address to thrombolysis as treatment choice in selected high risk patients with submassive PE. Guidelines also clarify the indications for unfractioned and low molecular weight heparins and fondaparinux. Therefore, in the present article, the authors focus on modern risk-based therapeutic guidelines of acute PE.
\end{abstract}

Keywords: Pulmonary embolism; Treatment; Prognosis; Biomarkers; Chocardiography; Hemodynamic; Guidelines

\section{Introduction}

Pulmonary embolism (PE) is a frequent disorder in

\footnotetext{
Manuscript accepted for publication March 19, 2009.

anternal Medicine, Cecina Hospital, Italy

${ }^{\mathrm{b}}$ Clinical Chemistry, Cecina Hospital, Italy

'Emergency Room, Cecina Hospital, Italy

${ }^{\mathrm{d} C a r d i o l o g y}$, Cecina Hospital, Italy

eRadiodiagnostics, cecina Hospita, Italy

Internal Medicine, Santa Maria Nuova Hospital, Florence, Italy

${ }^{\mathrm{g}}$ Thrombosis Center, University of Siena, Siena, Italy

${ }^{\text {h}}$ Corresponding author: UO Medicina Interna, Ospedale di Cecina, Professore a Contratto, Scuola di Specializzazione in Geriatria, Università di

Siena, Siena. Via Montanara, Localita Ladronaia, 57023, Cecina (Li), Italy.

E-mail: luca.masotti@tin.it
}

doi:10.4021/jocmr2009.03.1229 clinical practice with a hospital incidence of $0.4 \%$ of all admissions [1]. Mortality rate varies according to clinical presentation, in fact, it is about $2 \%$ in patients who appear normotensive without right heart compromise, it increases to $10-15 \%$ in normotensive patients with right heart compromise, and reaches around $30 \%$ in patients who present with cardiogenic shock and $70 \%$ in patients who present with cardiac arrest [2]. Treatment is usually effective when properly and quickly started; it should be kept in mind that about $10 \%$ of patients died within the first hour from onset of symptoms [2]. Thus early diagnosis, quick and appropriate treatment customized according to strict clinical and prognostic evaluation of the patient, are crucial moments in the modern therapeutic approach to this disease.

Since many years, thrombolysis was recognized to find indications in haemodinamically unstable patients with PE, in other words, in patients with shock (systolic blood pressure, $\mathrm{SBP}$, under $90 \mathrm{~mm} \mathrm{Hg}$ ). Up to now, the big question on therapy has been represented whether or not thrombolysis was also indicated in normotensive patients with evidence of right heart dysfunction (RHD) who have a significantly worse prognosis than normotensive patients without RHD. In the MAPPET III Study (Management Strategies And Prognosis of Pulmonary Embolism Trial-3), Konstantinides et al. had shown that, although in normotensive patients with RHD (condition defined as submassive-intermediate risk PE, see later), treatment with thrombolysis reduced mortality in a non-significant percentage, $3.4 \%$ in patients treated with unfractionated heparin (UFH) vs $2.2 \%$ in patients thrombolysed, $\mathrm{p}=\mathrm{ns}$, compared with more bleeding events in patients treated with thrombolysis; at the same time; thrombolytic treatment, added in patients initially treated with UFH with subsequent evolution toward hemodynamic instability (rescue treatment), clearly and significantly reduced mortality compared to patients not undergoing this treatment in the event of deteriorating hemodynamics (mortality $10.2 \mathrm{vs}$. $24.6 \%$ respectively, $\mathrm{p}<0.05$ ) [3]. Therefore, this trial had established the role of thrombolysis as rescue therapy in normotensive patients with RHD and initially treated with UFH that evolves towards hemodynamic instability, but thrombolysis was not recommended by the VII Edition guidelines of American College of Chest Physicians (ACCP) in nor- 


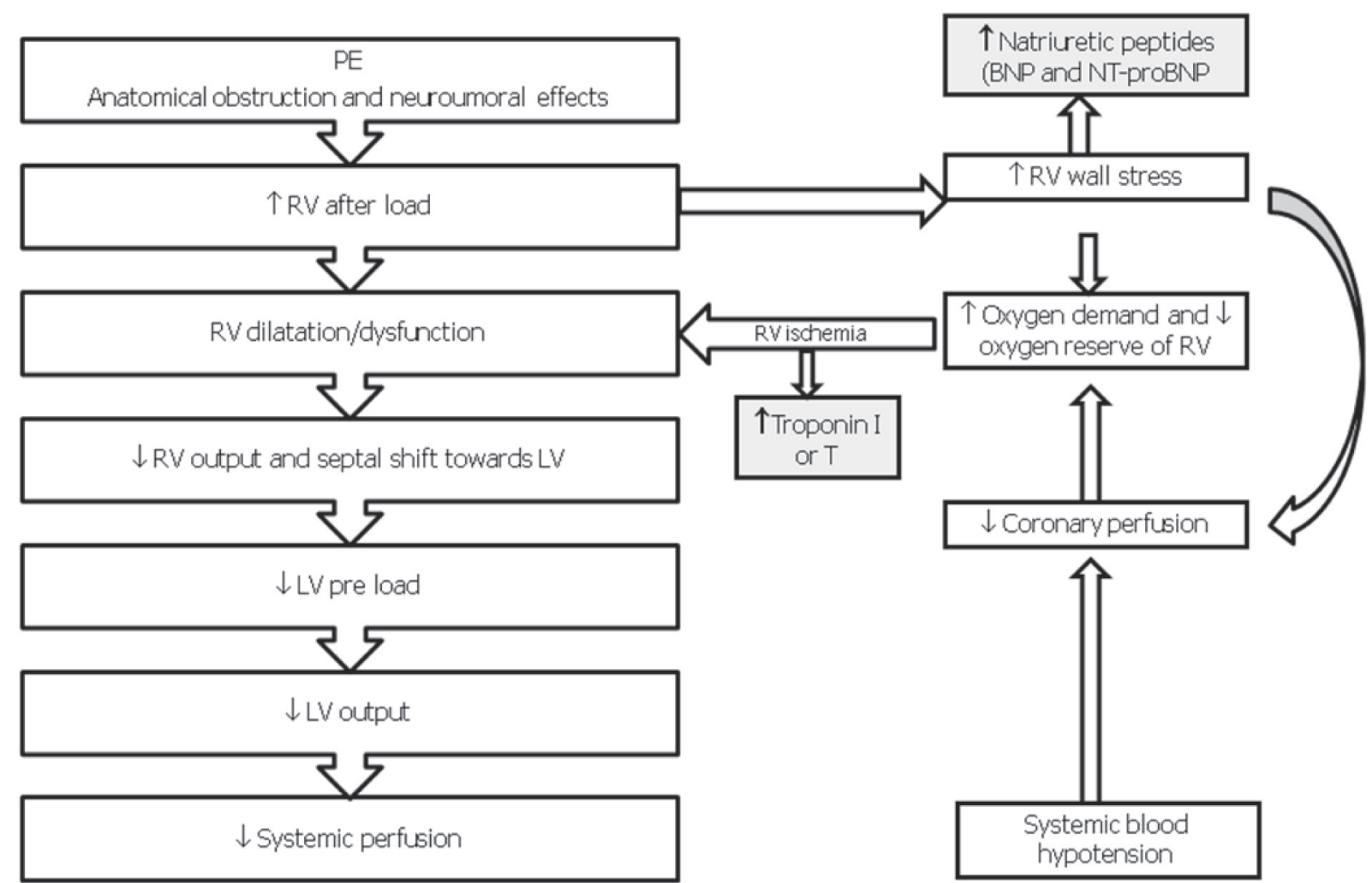

Figure 1. Pathophysiology of hemodynamic instability due to PE. RV, Right Ventricle; LV, Left Ventricle; BNP, Brain Natriuretic Peptide; NT-proBNP, AminoTerminal-proBrain Natriuretic Peptide.

motensive patients with RHD [4]. The increase of scientific evidence in favor of increased risk of mortality in patients with RHD without shock or hypotension at presentation and especially the considerable amount of evidence relating to the role of some prognostic biomarkers recently introduced (troponins and natriuretic peptides) complementary to the diagnostic imaging (echocardiography and computer tomography pulmonary angiography, CTPA) resulted in the development of new therapeutic guidelines of $\mathrm{PE}$ in the acute phase, based on the estimation of clinical risk and prognostic stratification much recently published (European Society of Cardiology, ESC, August 2008, ACCP, VIII Edition, June 2008), which will be discussed in this article $[5,6]$.

\section{Haemodinamic consequences of PE}

The extension of obstructed pulmonary arterial bed, the pre-existence of cardio-pulmonary disease and response to the release of vasoactive substances determined by local thrombus influence the pathophysiological response in acute PE $[2,7]$. The obstruction, caused by mechanical thrombus associated with pulmonary vasoconstriction determined by the release of vasoactive substances and hypoxia, leads to an increase of pulmonary vascular resistances and after-load of the right ventricle. This increase may result in right heart dilation, myocardial ischemia and hypokinesia, trcuspidal regurgitation or insufficiency, and finally right ventricular failure. In some patients, a rapid deterioration in progressive systemic arterial hypotension, cardiogenic shock and cardiac arrest may occur. Approximately 5-10\% of patients with normal blood pressure at presentation have a rapid deterioration in the early stages of the hospital admission, and this event is due to the recurrence of embolisation or to the acute dysfunction of the right ventricle [2, 7]. The hemodynamic consequences of PE are summarized in Figure 1. The respiratory consequences of $\mathrm{PE}$ are represented mainly by mismatch ventilation/perfusion (pulmonary areas ventilated but not perfused), increased of total dead space and right-left shunts $[2,7]$.

\section{Old and new clinical classification of PE}

$\mathrm{PE}$ is classically divided into massive form when manifested by cardiac arrest, cardiogenic shock or arterial hypotension (haemodynamically unstable form) form, submassive (PE with RHD but without hemodynamic instabil- 
Table 1. Clinical classification of PE

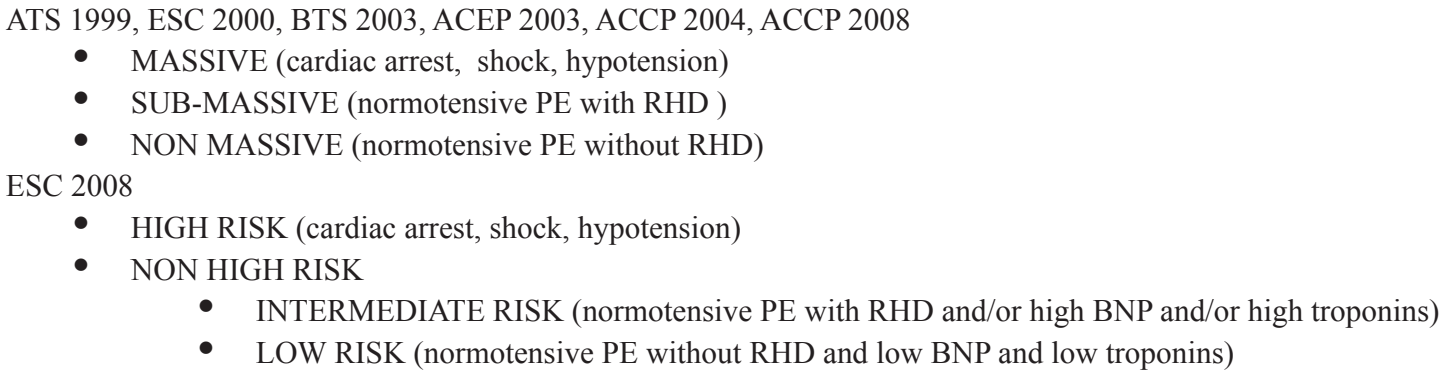

- MASSIVE (cardiac arrest, shock, hypotension)

- SUB-MASSIVE (normotensive PE with RHD )

- NON MASSIVE (normotensive PE without RHD)

ESC 2008

- HIGH RISK (cardiac arrest, shock, hypotension)

- NON HIGH RISK

- INTERMEDIATE RISK (normotensive PE with RHD and/or high BNP and/or high troponins)

- LOW RISK (normotensive PE without RHD and low BNP and low troponins)

ATS, American Thoracic Society; ESC, European Society of Cardiology; BTS, British Thoracic Society; ACEP; American College of Emergency Physicians; ACCP; American College of Chest Physicians; RHD; right heart dysfunction.

ity) and non-massive form (PE without RHD and without hemodynamic instability, usually caused by an obstruction of pulmonary arterial bed $<30 \%)[2,6,8,9]$.

Most recently, European Society of Cardiology (ESC) proposed to divide the clinical pictures of $\mathrm{PE}$ on the basis of clinical risk and prognosis; it follows that $\mathrm{PE}$ is divided into high risk (previous massive PE, mortality $>15 \%$ in the acute phase) and not high risk forms [5]. The latter is divided into intermediate risk form (previous sub-massive PE, mortality $3-15 \%$ ) and low-risk form (previous non-massive PE, mortality $<3 \%$ ). Table 1 summarizes the ESC classification of PE.

\section{Risk assessment and prognostic stratification}

Patients over 75 years, bed rest over four days, cancer, chronic obstructive pulmonary disease, heart failure, kidney failure, tachycardia and syncope are all clinical and comorbidity indicators of poor prognosis in acute PE $[10,11]$.

However the most important negative prognostic indicator in patients with $\mathrm{PE}$ is shock at presentation or during the acute phase of illness [2]. The ESC defines high risk PE as when it occurs with shock or arterial hypotension (SBP < $90 \mathrm{~mm} \mathrm{Hg}$ ); the relief of RHD and elevated biomarkers is not necessary to define the high risk [5]. It was recently introduced a parameter defined as shock index (ratio between heart rate in beats per minute and $\mathrm{SBP}$ in $\mathrm{mm} \mathrm{Hg}$ ), it is easy to apply in clinical practice. A value of shock index $>1$ correlates with the echocardiographic presence of RHD and increased pulmonary arterial systolic pressure (sPAP) and it is associated with increased hospital and thirty-days mortality $[12,13]$.

Pulmonary Embolism Severity Index (PESI) is a score

Table 2. ESC criteria for identifying the prognostic risk of PE

\begin{tabular}{llll}
\hline $\begin{array}{l}\text { Risk } \\
\text { (mortality in acute } \\
\text { phase \%) }\end{array}$ & Shock/hypotension & $\begin{array}{l}\text { Echocardiographic and } \\
\text { biomarkers findings of } \\
\text { RHD } \\
(\uparrow \text { BNP or NTpro-BNP) }\end{array}$ & $\begin{array}{l}\text { Findings of myocardial } \\
\text { injury: } \uparrow \text { troponin I or T }\end{array}$ \\
\hline $\begin{array}{l}\text { High } \\
(15 \%)\end{array}$ & Present & Present* & Present* \\
& & & \\
Intermediate & Absent & present \\
$(3-15 \%)$ & Absent & Present & Present \\
& Absent & Present & Absent \\
Low & & & Absent \\
$(<3 \%)$ & Absent & Absent & \\
\hline
\end{tabular}

*generally present but not necessary to define high risk 


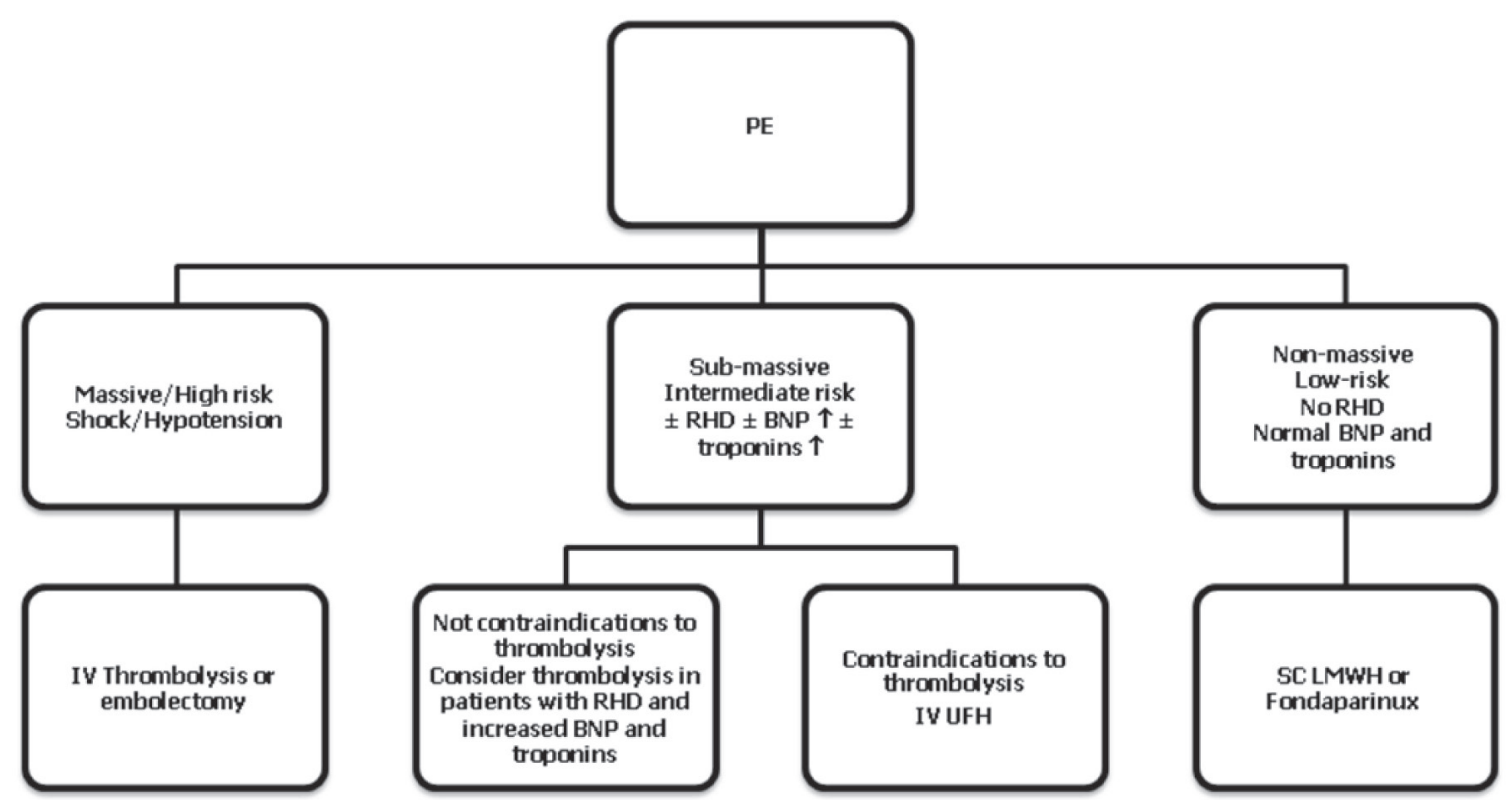

Figure 2. Summary of PE treatment according to modern guidelines. RHD, right heart dysfunction; BNP, brain natriuretic peptide; IV, intravenous; SC, subcutaneous.

that identifies 5 classes with a growing risk of mortality according to a strictly clinical parameters [14]. The patients in class I - II (low risk, mortality $\leq 1.2 \%$ ) may receive less aggressive therapy (subcutaneous low molecular weight heparins, LMWH, or fondaparinux and vitamin $\mathrm{K}$ antagonists) and they could be discharged from hospital more quickly or even could receive home treatment, whereas patients in class III - IV (mortality rate 4.8, 13.6, 25\%, respectively) should receive more aggressive treatment and close monitoring [14].

The electrocardiographic findings more indicative of negative prognosis in acute $\mathrm{PE}$ are the presence and number of negative $T$ waves on precordial leads [15]. The traditional parameters offered by arterial blood gas analysis do not seem to offer advantages in terms of prognosis.

Trans-thoracic echocardiography is the golden diagnostic standard for identifying RHD $[16,17]$. The main echocardiographic findings of RHD are represented by hypokinesia of right ventricle (mild, moderate, severe), dilatation of right ventricle (four chambers end-diastolic right ventricle/left ventricle diameter ratio $>1$ ), and findings of pulmonary arterial hypertension [17]. The presence of RHD at echocardiography is related to a negative prognosis both in shock patients and in normotensive patients $[11,17]$.

About $80 \%$ of patients with $\mathrm{PE}$ are normotensive at presentation; from one third to half of normotensive patients have echocardiographic RHD [10, 18]. In normotensive patients without RHD the range of hospital mortality is 0 - 9.6\%, while in patients with RHD is 11.8 - 23\% [19]. Normotensive patients with echocardiographic RHD have also reduced survival of 30 days (mortality increased to $17 \%$ ) compared to patients without RHD [18]. The presence of RHD at presentation correlates also with poor pulmonary thrombi dissolution at six months and with the greatest incidence of recurrence of venous thromboembolic events [20].

Computer tomography pulmonary angiography (CTPA) has become the golden standard diagnostic method for PE. It may give also prognostic information. By using information derived from CTPA imaging it is possible to consider 3 indices of PE severity: the CTPE index (Computer Tomography Pulmonary Embolism index), which assesses the degree of obstruction and the number of pulmonary arteries affected by PE [21]; the right ventricle/left ventricle diameter ratio assessed by CTPA, when increased more than 1, it seems to correlate with a negative prognosis [22]; and a new index much recently proposed by Ghanima et al, which assesses the degree of proximality of pulmonary arteries involvement in respect to main pulmonary artery [23]. All these indexes correlate with severity and prognosis of $\mathrm{PE}$, but in literature there are no consistent data for their use as a prognostic tool in routine clinical practice.

Among the biomarkers used for prognostic stratification of PE, with proven effectiveness are the results of troponins assay (I and $\mathrm{T}$ ) and natriuretic peptides (brain natriuretic peptide or its terminal portion, BNP and NT-proBNP respectively). The increase of troponins indicates myocardial damage and the expression of microinfarcts of right ventricular wall, while the natriuretic peptides are indices of RHD and their increase is attributable to stress of right ventricular wall [5]. The increase in these biomarkers was related to echocardiographic and CTPA data of RHD, it has negative predictive 
Table 3. Contraindications to thrombolysis in PE

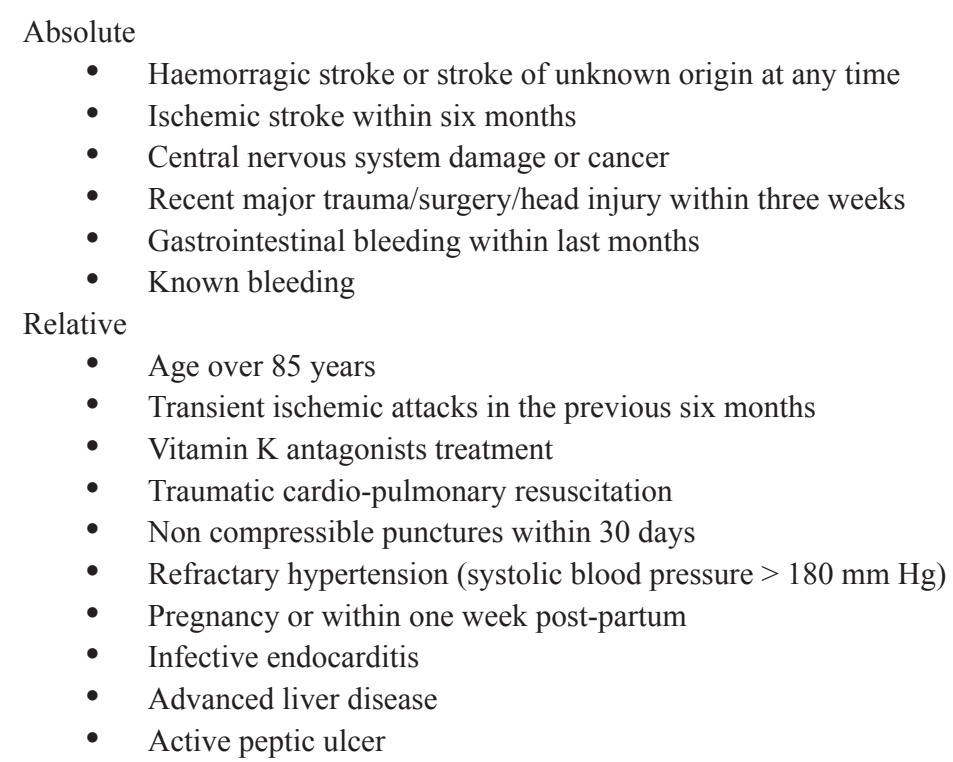

value [24]. Recent meta-analysis confirms that the increase in troponin I and $\mathrm{T}$ and natriuretic peptides is predictive of adverse prognosis in terms of mortality and morbidity in the acute phase of PE [25-27]. Hence, the rational basis for the introduction of these biomarkers is complementary to echocardiographic data in the evaluation of the clinical severity and prognostic stratification of PE made by ESC [5]. Table 2 summarizes the classes of risks suggested by ESC together with the parameters used to define them. It is possible to notice that the intermediate class at risk, for which therapy is most debated, provides for the possibility of three combinations arising from the presence or absence of RHD at echocardiography, increased natriuretic peptides and/or troponins.

Finally other biomarkers evaluated in terms of prognosis are D-dimer, its values seem to be correlated linearly with the commitment thrombotic proximal pulmonary artery and the clinical severity of PE [28], which, however, being very sensitive and relatively little specific, is unlikely as considerable prognostic index. The hearty type fatty acid binding proteins (htFABP) whose growth seems to correlate better than BNP and troponins with the prognosis of PE in the acute phase [29], but at the moment they are not widely disseminated in the hospital laboratories.

\section{Modern guidelines for antithrombotic therapy in the acute phase of $\mathrm{PE}$}

Modern treatment guidelines proposed by ESC and ACCP indicate that the treatment of acute PE is based on the clinical risk and prognostic stratification, it is based on pharmacological and non-pharmacological strategies. Figure 2 summarizes the antithrombotic treatment of PE depending on the category of risk.

First of all, patients with high probability of clinical PE should receive an intravenous bolus of UFH, less than contraindications, when they undergo inspection for confirmation.

In patients with massive/high-risk $\mathrm{PE}$ and hemodynamically unstable, thrombolytic therapy has shown to improve more quickly hemodynamic compromise, while it is uncertain about long-term outcomes [5, 6]. Among the possible thrombolytic agents, streptokinasis, urokinasis and recombinant tissue plasminogen activator (rtPA, alteplase), the last one is preferable. Schemes of thrombolytic therapy with infusions of short duration $(\leq 2 \mathrm{~h})$ are suggested compared to longer infusions $(12-24 \mathrm{~h})$ that do not have beneficial effects on hemodynamics and that are burdened by higher bleeding complications. The rtPA is administered by bolus of $10 \mathrm{mg} \mathrm{IV}$, followed by 2 hours in a dose determined by body weight; it must not be exceeded the total dose of 100 $\mathrm{mg}$. In cases of extreme severity, it can be administered by dose of $0.6 \mathrm{mg} / \mathrm{kg}$ of bolus rtPA IV in rapid $5-15$ minutes, but not exceeding the maximum dose of $50 \mathrm{mg}$ IV. It should be noted that during the infusion of rtPA, The UFH infusion should be discontinued and then resumed after the infusion of thrombolytic $[5,6]$. 
Thrombolytic treatment is indicated (recommendation IIB) in selected patients with sub-massive PE/intermediate risk who have evidence of RHD echocardiographically and by biomarkers (BNP or NTproBNP), myocardial damage shown by increased levels of troponin I or T $[5,6]$ and not absolute contraindications to thrombolysis (Table 3 ).

Intravenous UFH should be reserved in patients with sub-massive PE/intermediate risk where there are contraindications to thrombolysis. A bolus of IV UFH (in the vast majority of cases are made 5000 IU IV bolus, in elderly patients might be prudent a bolus of $3000 \mathrm{IU}$ ) followed by continuous infusion of UFH with infusion pump or syringe pump in a dose would lead to an increase of aPTT (time of activated partial thromboplastin) of $1.5-2.5$ times the normal value, monitored by the aPTT nomograms $[5,6]$.

In patients with non-massive/low-risk PE, the subcutaneous LMWH or fondaparinux at anticoagulant dose are recommended (example enoxaparin $100 \mathrm{IU} / \mathrm{kg}$ twice daily, $5 \mathrm{mg}$ fondaparinux if weight $<50 \mathrm{Kg}, 7.5 \mathrm{mg}$ if weight between 50 and $100 \mathrm{~kg}, 10 \mathrm{mg}$ if weight $>100 \mathrm{~kg}$ in single dose). LMWH should be avoided in patients with renal failure and/ or severe obesity; in the first situation the choice is UFH, in obese patients the choice is subcutaneous fondaparinux at dosage of $10 \mathrm{mg} /$ day. LMWH should be continued for the first 3 - 6 months for prevention of secondary venous thromboembolism in patients with cancer. Fondaparinux is further indication in patients with heparin induced thrombocytopenia. The intravenous UFH, LMWH and fondaparinux should be administered at least for five consecutive days, unless complications occurring, overlapping vitamin $\mathrm{K}$ antagonists (VKA) $[5,6]$.

VKA should be started in the same day of heparins or fondapariunx, overlapping it, with the aim of achieving a therapeutic range to maintain the value of INR (International Normalized Ratio) in the range 2 - 3 (target 2.5) in most patients. Heparins or fondaparinux should be discontinued when the value of INR is included in the therapeutic range for at least two consecutive days $[5,6]$.

The indication for the positioning of vena cava filters are limited to situations where VKA are absolutely contraindicated or VKA should be discontinued for the appearance of complications, or if PE has occurred in patients under VKA well conducted (INR in the therapeutic range). Vena cava filter could find indication in patients undergoing pulmonary embolectomy or thromboendoarterectomy for chronic thromboembolic pulmonary hypertension $[5,6]$.

Surgical or mechanical embolectomy is indicated in patients with massive/high risk PE, where there are absolute contraindications to thrombolysis, or this was ineffective after documentation of pulmonary thrombus through transesophageal echocardiogram or pulmonary arteriography. Pulmonary thromboendoarterectomy is indicated in chronic tromboembolic pulmonary hypertension $[5,6]$.

\section{Conclusions}

PE is a common disease in clinical practice, burdened by high morbidity and mortality especially if combined with hemodynamic instability. Modern guidelines based on the risk estimate according to clinical and instrumental indicators and biomarkers can customize treatment with a good chance of success, with minimum complications. The most important news on treatment is the possibility of thrombolysis in selected normotensive patients with echocardiographic and laboratory findings which are at high risk of adverse prognosis. This strategy will be evaluated in prospective clinical multicenter intervention trials which will determine their effectiveness. The current trial undergoing is an important European trial (PEITHO Study) whose results are expected in the coming years and that will give important answers to the previous question [30].

\section{Conflict of Interest}

The authors declare no conflicts of interest.

\section{References}

1. Stein PD, Beemath A, Olson RE. Trends in the incidence of pulmonary embolism and deep venous thrombosis in hospitalized patients. Am J Cardiol 2005;95:1525-1526.

2. Wood KE. Major pulmonary embolism: review of a pathophysiologic approach to the golden hour of hemodynamically significant pulmonary embolism. Chest 2002;121:877-905.

3. Konstantinides S, Geibel A, Heusel G, Heinrich F, Kasper W. Heparin plus alteplase compared with heparin alone in patients with submassive pulmonary embolism. N Engl J Med 2002;347:1143-1150.

4. Buller HR, Agnelli G, Hull RD, Hyers TM, Prins MH, Raskob GE. Antithrombotic therapy for venous thromboembolic disease: the Seventh ACCP Conference on Antithrombotic and Thrombolytic Therapy. Chest 2004;126:401S-428S.

5. Torbicki A, Perrier A, Konstantinides S, Agnelli G, Galie N, Pruszczyk P, Bengel F, et al. Guidelines on the diagnosis and management of acute pulmonary embolism: the Task Force for the Diagnosis and Management of Acute Pulmonary Embolism of the European Society of Cardiology (ESC). Eur Heart J 2008;29:2276-2315.

6. Kearon C, Kahn SR, Agnelli G, Goldhaber S, Raskob GE, Comerota AJ. Antithrombotic therapy for venous thromboembolic disease: American College of Chest Physicians Evidence-Based Clinical Practice Guidelines (8th Edition). Chest 2008;133:454S-545S.

7. Goldhaber SZ, Elliott CG. Acute pulmonary embolism: 
part I: epidemiology, pathophysiology, and diagnosis. Circulation 2003;108:2726-2729.

8. British Thoracic Society guidelines for the management of suspected acute pulmonary embolism. Thorax 2003;58:470-483.

9. Clinical policy: critical issues in the evaluation and management of adult patients presenting with suspected pulmonary embolism. Ann Emerg Med 2003;41:257-270.

10. Becattini C, Agnelli G. Acute pulmonary embolism: risk stratification in the emergency department. Intern Emerg Med 2007;2:119-129.

11. Goldhaber SZ, Visani L, De Rosa M. Acute pulmonary embolism: clinical outcomes in the International Cooperative Pulmonary Embolism Registry (ICOPER). Lancet 1999;353:1386-1389.

12. Toosi MS, Merlino JD, Leeper KV. Prognostic value of the shock index along with transthoracic echocardiography in risk stratification of patients with acute pulmonary embolism. Am J Cardiol 2008;101:700-705.

13. Otero R, Trujillo-Santos J, Cayuela A, Rodriguez C, Barron M, Martin JJ, Monreal M. Haemodynamically unstable pulmonary embolism in the RIETE Registry: systolic blood pressure or shock index? Eur Respir J 2007;30:1111-1116.

14. Aujesky D, Perrier A, Roy PM, Stone RA, Cornuz J, Meyer G, Obrosky DS, et al. Validation of a clinical prognostic model to identify low-risk patients with pulmonary embolism. J Intern Med 2007;261:597-604.

15. Geibel A, Zehender M, Kasper W, Olschewski M, Klima C, Konstantinides SV. Prognostic value of the ECG on admission in patients with acute major pulmonary embolism. Eur Respir J 2005;25:843-848.

16. Goldhaber SZ. Echocardiography in the management of pulmonary embolism. Ann Intern Med 2002;136:691700 .

17. Kreit JW. The impact of right ventricular dysfunction on the prognosis and therapy of normotensive patients with pulmonary embolism. Chest 2004;125:1539-1545.

18. Kucher N, Rossi E, De Rosa M, Goldhaber SZ. Prognostic role of echocardiography among patients with acute pulmonary embolism and a systolic arterial pressure of $90 \mathrm{~mm} \mathrm{Hg}$ or higher. Arch Intern Med 2005;165:17771781.

19. Gibson NS, Sohne M, Buller HR. Prognostic value of echocardiography and spiral computed tomography in patients with pulmonary embolism. Curr Opin Pulm Med 2005;11:380-384.

20. Kaczynska A, Kostrubiec M, Pacho R, Kunikowska J,
Pruszczyk P. Elevated D-dimer concentration identifies patients with incomplete recanalization of pulmonary artery thromboemboli despite 6 months anticoagulation after the first episode of acute pulmonary embolism. Thromb Res 2008;122:21-25.

21. Qanadli SD, El Hajjam M, Vieillard-Baron A, Joseph T, Mesurolle B, Oliva VL, Barre O, et al. New CT index to quantify arterial obstruction in pulmonary embolism: comparison with angiographic index and echocardiography. AJR Am J Roentgenol 2001;176:1415-1420.

22. Quiroz R, Kucher N, Schoepf UJ, Kipfmueller F, Solomon SD, Costello P, Goldhaber SZ. Right ventricular enlargement on chest computed tomography: prognostic role in acute pulmonary embolism. Circulation 2004;109:2401-2404.

23. Ghanima W, Abdelnoor M, Holmen LO, Nielssen BE, Sandset PM. The association between the proximal extension of the clot and the severity of pulmonary embolism (PE): a proposal for a new radiological score for PE. J Intern Med 2007;261:74-81.

24. Binder L, Pieske B, Olschewski M, Geibel A, Klostermann B, Reiner C, Konstantinides S. N-terminal probrain natriuretic peptide or troponin testing followed by echocardiography for risk stratification of acute pulmonary embolism. Circulation 2005;112:1573-1579.

25. Becattini C, Vedovati MC, Agnelli G. Prognostic value of troponins in acute pulmonary embolism: a meta-analysis. Circulation 2007;116:427-433.

26. Klok FA, Mos IC, Huisman MV. Brain-type natriuretic peptide levels in the prediction of adverse outcome in patients with pulmonary embolism: a systematic review and meta-analysis. Am J Respir Crit Care Med 2008; 178:425-430.

27. Coutance G, Le Page O, Lo T, Hamon M. Prognostic value of brain natriuretic peptide in acute pulmonary embolism. Crit Care 2008;12:R109.

28. De Monye W, Sanson BJ, Mac Gillavry MR, Pattynama PM, Buller HR, van den Berg-Huysmans AA, Huisman MV. Embolus location affects the sensitivity of a rapid quantitative D-dimer assay in the diagnosis of pulmonary embolism. Am J Respir Crit Care Med 2002;165:345-348.

29. Puls M, Dellas C, Lankeit M, Olschewski M, Binder L, Geibel A, Reiner C, et al. Heart-type fatty acid-binding protein permits early risk stratification of pulmonary embolism. Eur Heart J 2007;28:224-229.

30. Peitho. Pulmonary embolism thrombolysis trial. NCT00639743. www.clinicaltrials.gov 